\title{
Exobronchial Pulmonary Eye: Transthoracic Ultrasound- Guided Transbronchial Lung Biopsy in Different Pulmonary Shadows
}

\author{
Ahmed Mohammed Abumossalam ${ }^{\mathbf{1}}$, Taha Taha Abdelgawad ${ }^{\mathbf{1}, \text { Amro Abdelhamid Moawd }}{ }^{\mathbf{1}}$, \\ Abdelhadi Mohammed Shebl ${ }^{2}$ \\ ${ }^{1}$ Thoracic medicine department, Faculty of medicine, Mansoura University, Mansoura, Egypt \\ ${ }^{2}$ Pathology department, Faculty of medicine, Mansoura University, Mansoura, Egypt
}

Email address:

abumossalam79@yahoo.com (A. M. Abumossalam)

To cite this article:

Ahmed Mohammed Abumossalam, Taha Taha Abdelgawad, Amro Abdelhamid Moawd, Abdelhadi Mohammed Shebl. Exobronchial Pulmonary Eye: Transthoracic Ultrasound-Guided Transbronchial Lung Biopsy in Different Pulmonary Shadows. International Journal of Medical Imaging. Vol. 3, No. 3, 2015, pp. 41-48. doi: 10.11648/j.jimi.20150303.11

\begin{abstract}
Background: Transbronchial lung biopsy (TBLB) is one of the most imperative applications of flexible bronchoscopy that may preclude the need for an open lung biopsy. A real-time ultrasonographic guidance may increase the diagnostic yield of TBLB. Purpose: The aim of this study is to evaluate the diagnostic yield of a real-time transthoracic ultrasound-guided transbronchial lung biopsy (TTUS-TBLB) in different pulmonary shadows. Patients and methods: One hundred and seventeen patients with radiological pulmonary shadows were submitted for fiberoptic bronchscopy and underwent either conventional TBLB (cTBLB) that only supported by prior localization with chest computed tomography (CT) in 84 patients (group 1) or under a real-time TTUS-TBLB in 33 patients (group 2). Results: The overall diagnostic yield of TBLB was $74.4 \%$ ( 87 cases out of $117 ; 79$ cases by the first TBLB and 8 cases by second TBLB). The final diagnosis of the enrolled patients was; fifty two cases proved to be malignant (42 cases diagnosed by TBLB) and sixty five cases proved to be nonmalignant (45 cases diagnosed by TBLB). TTUS-TBLB achieved diagnosis in $81.81 \%$ of cases and cTBLB achieved diagnosis in $71.43 \%$. Conclusion: The results of real-time TTUS-TBLB were promising and may improve the overall diagnostic yield of TBLB in either benign or malignant pulmonary shadows.
\end{abstract}

Keywords: TBLB, Transthoracic Ultrasound, Pulmonary Shadows, Mass, Cavity

\section{Introduction}

The flexible bronchoscope was initiated to be used in the United States late 1960s and had given pulmonologists access to the lung as never before, $[1,2]$ The small biopsy samples obtained with this bronchoscope present a unique challenge to the general pathologist, not the least of which is knowledge of the clinical and radiologic background that prompted the biopsy, [3, 4]

Biopsy from lung tissue is required for appropriate diagnosis and management of a variety of pulmonary disorders. Samples of lung tissue for diagnostic purpose may be obtained through the transbronchial lung biopsy (TBLB), computed tomography-guided fine needle aspiration (CTFNA), and video-assisted thoracoscopic or surgical lung biopsy. With few exceptions, surgical lung biopsy is the gold standard for attaining lung tissue but it is invasive, costly, requires general anesthesia and hospital admission. The application of CT-FNA is largely limited to peripheral pulmonary lesions. The specimen from CT-FNA is generally small sized and insufficient for tissue diagnosis of benign disease processes. Pneumothorax can develop in up to a quarter and chest tube is required in about $10 \%$ of patients after CT-FNA, [5] Quite the reverse, TBLB has wider clinical applications, the complication rate is low and the procedure can safely be performed in an outpatient bronchoscopy suite under conscious sedation. Serial lung biopsies are also best obtained with the transbronchial approach. Several large case series have taken in hand the diagnostic yield of TBLB in miscellaneous groups of patients with a diversity of 
underlying disease processes, [6-11].

Open lung biopsy, although the standard for definitive diagnostic yield, involves formidable patient morbidity related to chest tubes placement and postoperative pain. Transbronchial forceps lung biopsy has been emphasized as an alternative method for biopsy, [12]. Moreover, several authors have documented the feasibility of obtaining pulmonary parenchyma for pathological examination through the flexible fiberoptic bronchoscope, $[13,14]$.

Even supposing TBLB provided adequate lung specimen in 70-90\% of cases, a specific diagnosis could not be rendered in a significant proportion of the patients. The overall diagnostic yield for specific diagnosis varies widely, depending on the size, location and extent of lung infiltrates, and the nature of underlying lung disease. The results of TBLB also depend on the experience and technical skills of the bronchoscopic operator. Care is warranted in interpreting the literature on diagnostic yield of TBLB because most authors have included nonspecific fibrosis or organizing pneumonia in the diagnostic yield and only a few have applied more stringent criteria for pathologic interpretation of TBLB specimens. Nonspecific findings on TBLB are often unhelpful and can lead to erroneous clinical decisions, [15]

TBLB is generally safe, although severe and lives threatening procedure-related complications are occasionally encountered. According to a recent nationwide survey from Japan, the complication rate after forceps biopsy for a peripheral pulmonary lesion was $1.79 \%$. [16] In comparison, a review of 173 procedures from a university hospital has reported a complication rate of $6.8 \%$ after TBLB, [17] Two most common complications of TBLB are bleeding and pneumothorax. The reported incidence of bleeding after TBLB has varied from 0 to $26 \%$ in different series. Serious bleeding occurs in 1-2 \% of patients undergoing TBLB, [18, 19] The risk of bleeding is significantly higher in immunocompromised patients and in patients with underlying renal insufficiency, [20, 21, 22] Reports of deaths due to uncontrolled bleeding after TBLB are exceedingly uncommon, but it is plausible that it is under-reported. In the Japanese survey mentioned above, $0.85 \%$ of 57,199 patients experienced significant bleeding after TBLB, but there were no deaths, [16]. Therefore, expanding the endoscopist's view beyond the airways is essential as in endobronchial ultrasound. So this study aims to evaluate the diagnostic yield of a real-time transthoracic ultrasound guided trasnbronchial lung biopsy (TTUS-TBLB) in different pulmonary shadows.

\section{Patients and Methods}

\subsection{Methods}

This prospective experimental study (clinical trial) conducted over 117 patients with radiological pulmonary shadows close to chest wall submitted for fiberoptic bronchscopy and either underwent conventional transbronchial lung biopsy (cTBLB) [only supported by prior localization of the lesion with chest computed tomography
(CT)] in 84 patients (group 1) or under a real-time transthoracic ultrasound-guided transbronchial lung biopsy (TTUS-TBLB) (using Mindray DP 2200 with convex two dimensional probe of low frequency $2.5-5 \mathrm{MHz}$ ) in 33 patients (group 2). The patients were excluded from the study if they have contraindications to fiberoptic bronchoscopy, bleeding diathesis or presence of endobronchial lesions. This study was performed in Mansoura university hospitals chest department during the period from June 2013 to January 2015

\subsection{Procedures}

TBLB were carried out using fiberoptic bronchoscopy (Pentax FB 19 TV; Tokyo, Japan) under local anesthesia. Topical anesthesia was induced using lidocaine 5\% spray and nebulized form $5 \mathrm{ml}$ distilled in $3 \mathrm{ml}$ normal saline in addition to conscious sedation using propofol $(1-2 \mathrm{mg} / \mathrm{kg})$. Anesthesia was maintained with intravenous propofol (4 to 6 $\mathrm{mg} / \mathrm{kg} / \mathrm{h}$ ) according to the hemodynamic parameters of the patients and according patient need. TBLBs were performed blindly in 84 patients (only supported by pre-bronchoscopy $\mathrm{CT}$ chest localization of the lesion) and real-time transthoracic ultrasonography guidance in 33 patients (using Mindray DP 2200) with two dimensional ultrasound probe of low frequency $2.5-5 \mathrm{MHz}$ ) for localization of the lesion. Concomitant application of transthoracic ultrasound probe with adjusted insinuation in the intercostal spaces with advancement of FOB after passing the vocal cords for actual visualization of bronchoscopy shaft through airways that appears as echogenic line till attaining the prebronchoscopy localization of the site pulmonary shadow to be biopsied. The FOB shaft then moved forward till reach the site of pulmonary lesion then actual simultaneous forceps (Mako biopsy forceps with diameter; $2.4 \mathrm{~mm}$ and length; $230 \mathrm{~cm}$ ) progression till contacting segmental pulmonary tissue affected with close seizing of specimen. Ultrasound can visualize forceps biopsy handling in cases of sizable mass/s or solid compact lesion but with limited access in small cavernous lesions. For each patient a number of at least 3 TBLB specimens were obtained in one session. In all cases; the specimens were stained by Haematoxylin \& Eosin for cytopathological examination, in addition to some special stains like Periodic Acid Shief PAS and Ziehl Neelsen ZN.

TBLB was considered unhelpful and negative when the procedure failed to obtain lung tissue, or failed to obtain a characteristic histopathologic pattern (normal tissue). Complications related to bronchoscopic procedures were routinely recorded and were properly managed. They were reviewed for evidence of: endobronchial hemorrhage, iatrogenic pneumothorax, severe respiratory failure (oxygen saturation $<85 \%$ and/or need of assisted ventilation), and cardiac arrhythmias, or cardiac arrest during or immediately after the bronchoscopic procedures. Cases developed pneumothorax was properly managed by intercostal tube placement for 2-5 days and cases complicated by haemoptysis were controlled by haemostatic. For cases not diagnosed by first TBLB, either second TBLB by the same 
guidance, percutaneous CT guided biopsy were done. Patients follow up was considered as a diagnostic modality.

\subsection{Statistical Analysis}

Data was analyzed using SPSS (Statistical Package for Social Sciences) version 21. Qualitative data was presented as number and percentage. Quantitative data was presented

for normality by Kolmogrov-Smirnov test. Normally distributed data was presented as mean and standard deviation. Comparison between groups was done using Chisquare test. $\mathrm{P}$ value $<0.05$ was considered significant.

\section{Results}

Table (1). Demographic data and radiological pattern in the studied population.

\begin{tabular}{|c|c|c|c|}
\hline & Malignant group No $=(52)$ & Nonmalignant group $\mathrm{No}=(65)$ & Total (117) \\
\hline Age (mean $\pm \mathrm{SD})$ & $50.55( \pm 13.612)$ & $47.41( \pm 11.488)$ & $48.54( \pm 12.327)$ \\
\hline \multicolumn{4}{|l|}{ Sex } \\
\hline Female & 22 & 25 & 47 \\
\hline Total & (52) $44.45 \%$ & (65) $55.56 \%$ & 117 \\
\hline \multicolumn{4}{|l|}{ Radiological Pattern } \\
\hline Cavity & $1(2.4 \%)$ & $2(2.7 \%)$ & 3 \\
\hline Infiltrate & $15(35.7 \%)$ & $48(64 \%)$ & 63 \\
\hline Mass & $21(50 \%)$ & $7(9.3 \%)$ & 28 \\
\hline Mediastinal lymphadenopathy & $0(0 \%)$ & $1(1.3 \%)$ & 1 \\
\hline Mediastinal lymphadenopathy and infiltrate & $0(0 \%)$ & $1(1.3 \%)$ & 1 \\
\hline$S P N$ & $1(2.4 \%)$ & $0(0 \%)$ & 1 \\
\hline Nodules & $2(4.8 \%)$ & $8(10.7 \%)$ & 10 \\
\hline Patch & $1(2.4 \%)$ & $7(9.3 \%)$ & 8 \\
\hline Patch + mass & $1(2.4 \%)$ & $1(1.3 \%)$ & 2 \\
\hline
\end{tabular}

-SPN [solitary pulmonary nodule]- SD [Standard deviation] - No [Number]

As shown in table (1) according to final diagnosis of 117 cases they were subdivided into malignant group that comprised 52 cases $(44.45 \%)$ and nonmalignant group that comprised 65 cases $(55.56 \%)$. The mean age of studied cases was 48.54 year \pm 12.327 . The mean age of malignant group was 50.55 year \pm 13.612 while for nonmalignant group was 47.41 year \pm 11.488 . From table (1) infiltrate was the predominant pattern encountered in nonmalignant group (64\%) followed by nodules $(10.7 \%)$ then mass and patch (9.3\% for each) while in malignant group mass was the predominant pattern $(50 \%)$ followed by infiltrate $(35.7 \%)$ then nodules $(4.8 \%)$ and lastly patch and patch with mass (2.4\% for each).

Table (2). Diagnosis of the studied cases (first TBLB).

\begin{tabular}{lll}
\hline Final diagnosis & Frequency & Percent \\
\hline 1-Malignant group & 36 & $30.76 \%$ \\
Adenocarcinoma & 10 & 8.5 \\
Adenosquamous carcinoma & 1 & 0.9 \\
Bronchioloalveolar carcinoma & 2 & 1.7 \\
Carcinoid tumour & 1 & 0.9 \\
Large cell carcinoma & 2 & 1.7 \\
Papillary adenocarcinoma & 1 & 0.9 \\
Pneumocyte hyperplasia & 3 & 2.6 \\
Small cell carcinoma & 2 & 1.7 \\
Squamous cell carcinoma & 12 & 10.3 \\
Squamous metaplasia & 2 & 1.7 \\
2-Nonmalignant group & 43 & $36.75 \%$ \\
Bronchial sclerosis. & 1 & 0.9 \\
Idiopathic pulmonary heamosedrosis & 1 & 0.9 \\
pneumonia & 24 & 20.5 \\
Invasive aspergillosis & 1 & 0.9 \\
Interstitial Pulmonary Fibrosis & 3 & 2.6 \\
Sarcoidosis. & 2 & 1.8 \\
Pulmonary alveolar proteinosis & 2 & 1.7 \\
Pulmonary congestion with heart failure cells. & 1 & 0.9 \\
\hline
\end{tabular}

\begin{tabular}{lll}
\hline Pulmonary tuberculosis & 8 & 6.8 \\
3- Negative biopsy & 38 & $32.47 \%$ \\
Total & 117 & 100.0 \\
\hline
\end{tabular}

As shown in table (2) from the first TBLB sample; malignant group comprised $30.76 \%$ and nonmalignant group comprised $36.75 \%$ of total studied cases. First sample failed to obtain diagnosis in $32.47 \%$ of cases. Squamous cell carcinoma accounted for $10.3 \%$ and adenocarcinoma accounted for $8.5 \%$ followed by small cell carcinoma, large cell carcinoma and bronchioloalveolar carcinoma presented $1.7 \%$ for each while adenosquamous carcinoma , carcinoid tumour and papillary adenocarcinoma showed $0.9 \%$ for each. In the nonmalignant group, pneumonia represented $20.5 \%$ followed by pulmonary tuberculosis $(6.8 \%)$. First TBLB succeeded in the diagnosis of 79 cases out of 117 cases $(67.52 \%)$ and failed in 38 cases $(32.47 \%)$.

Table (3). Overall diagnostic yield of cTBLB versus TTUS-TBLB in the studied cases.

\begin{tabular}{|c|c|c|c|}
\hline & $\begin{array}{l}\text { cTBLB } \\
\text { No=84 }\end{array}$ & $\begin{array}{l}\text { TTUS-TBLB } \\
\text { No=33 }\end{array}$ & Total \\
\hline \multicolumn{4}{|l|}{ First sample } \\
\hline 1-Malignant lesions & 30 & 6 & 36 \\
\hline 2-Beign lesions & 26 & 17 & 43 \\
\hline 3-Negative & 28 & 10 & 38 \\
\hline $\begin{array}{l}\text { Diagnostic Accuracy after } \\
1^{\text {st }} \text { session }\end{array}$ & $66.7 \%$ & $69.69 \%$ & $67.52 \%$ \\
\hline Second sample & 4 & 4 & 8 \\
\hline 1-Malignant lesions & 4 & 2 & 6 \\
\hline 2-Beign lesions & 0 & 2 & 2 \\
\hline 3-Negative & 0 & 0 & 0 \\
\hline Accuracy after $2^{\text {nd }}$ session & $71.43 \%$ & $81.81 \%$ & $74.4 \%$ \\
\hline
\end{tabular}

As shown in table (3) from the first session, the transthoracic US guided TBLB succeed in the diagnosis of 
$69.69 \%$ of cases underwent the procedure on the other hand blind TBLB succeed in the diagnosis of $66.7 \%$. However, by the second session, transthoracic US guided TBLB achieved diagnosis in $81.81 \% \%$ and blind TBLB achieved the diagnosis in $71.43 \%$. The overall diagnostic yield of TBLB was $74.4 \%$ ( 87 cases out of $117 ; 79$ cases by the first TBLB and 8 cases by second TBLB).

Table (4). Final diagnosis in cases with negative first TBLB.

\begin{tabular}{lll}
\hline Follow up & No \&\% & Final diagnosis \\
\hline Improvement with nonspecific & $18(47.4 \%)$ & Pneumonia \\
antimicrobial therapy & $2(5.3 \%)$ & Pulmonary Tuberculosis \\
& $6(15.78 \%)$ & Squamous cell carcinoma \\
CT guided True cut biopsy & $4(10.6 \%)$ & Hodgkin lymphoma \\
& $6(15.78 \%)$ & Adenocarcinoma \\
Second TBLB & $2(5.3 \%)$ & Sarcoidosis \\
\hline
\end{tabular}

Of the 38 negative biopsies pneumonia accounted for $47.4 \%$ and pulmonary tuberculosis and sarcoidosis showed $5.3 \%$ for each. Follow up of these cases as shown in table (4) was considered for two weeks; 18 cases proved to be inflammatory consolidation and improved with nonspecific antimicrobial treatment (4 cases in TTUS TBLB group and 14 in cTBLB). Twelve patients underwent CT guided true cut biopsy (10 in cTBLB and 2 in TTUS TBLB) and proved to be: 2 cases due to pulmonary TB, 6 cases due to squamous cell carcinoma and 4 cases due to pulmonary Hodgkin lymphoma ( 2 cases in TTUS TBLB group and ). On the third hand 6 cases diagnosed to be adenocarcinoma and 2 cases due to sarcoidosis by a second TBLB session ( 4 cases for each group by the same guidance).

Table (5). Complications of TBLB in studied cases.

\begin{tabular}{llll}
\hline Complication & cTBLB & TTUS-TBLB & Total \\
\hline Residual pneumothorax & 2 & 1 & $2.56 \%$ \\
Haemoptysis & 1 & 1 & $1.7 \%$ \\
Malignant group & 2 & 1 & $2.56 \%$ \\
Nonmalignant group & 1 & 1 & $1.7 \%$ \\
Total & 3 & 2 & $4.2 \%$ \\
\hline
\end{tabular}

As shown in table (5) out of the 117 cases 5 cases developed complications (4.2\%) that were in the form of residual pneumothorax in 3 patients, $2.56 \%$ ( 2 in malignant group and one in nonmalignant group) and haemoptysis in two patients $1.7 \%$ (one in each group) that was controlled by haemostatics and did not need surgical intervention. No complications were detected from manipulation of negative cases either by CT guided biopsy or rebiopsy by TBLB. No cases of mortality was recorded either during or postprocedure within 1 week. cTBLB presented $2.56 \%$ of cases while TTUS-TBLB accounted for $1.7 \%$ of complications encountered.

\section{Discussion}

Transbronchial lung biopsy is an essential endoscopic skill for every bronchoscopist that is usually performed in outpatient setting under conscious sedation. A successful
TBLB may obviate need for surgical lung biopsy, which is more invasive and needs general anesthesia. The commonest indication of TBLB is to obtain biopsy specimen from different pulmonary lesions. Accurate localization and handling of lung lesions needs precise supervision that varies according site, level of equipments availability, type of the patients and operator proficiency. Many assistant radiological tools had been emerged instigating from fluoroscopy, CT chest guidance come to an end with endobronchial sonohgraphy. Transthoraic ultrasonography guided biopsy has brought to be a promising tool being cheap, rapid, accessible, does not need complex skill and difficult training.

In a study conducted by Lai et al., 1996, [23], of 170 patients entered into the study, there were 120 cases of pulmonary malignancy (including 37 squamous cell carcinoma, 68 adenocarcinoma, 4 small cell carcinoma, 6 alveolar cell carcinoma, 2 large cell carcinoma, and 3 metastatic cancer in the lungs) and 50 cases of benign pulmonary lesions (including 40 pulmonary tuberculosis, six localized pneumonia, two organizing pneumonia, one cryptococcosis, and one carcinoid). Of the remaining 36 patients in whom bronchoscopy gave negative results, 13 were diagnosed by chest CT-guided biopsy and 23 were diagnosed by exploratory thoracotomy. The data from this study also confirm that the percentage of positive diagnosis correlates with the diameter of the solitary pulmonary nodule (SPN) in both cancer and TB patients. If the histological findings showed 'acute and chronic inflammation', the sample was excluded as diagnostic, since this cannot rule out malignancy. In the literature, flexible bronchoscopy with TBLB, brushing and washing has a $10-28 \%$ yield for malignant nodules less than $2 \mathrm{~cm}$ in diameter, and $40-80 \%$ for malignant nodules greater than $2 \mathrm{~cm}$ in diameter, [24, 25]. In our study; the first TBLB sample malignant group comprised $30.76 \%$ (squamous cell carcinoma accounted for $10.3 \%$ and adenocarcinoma accounted for $8.5 \%$ followed by small cell carcinoma, large cell carcinoma and bronchioloalveolar carcinoma presented $1.7 \%$ for each while adenosquamous carcinoma, carcinoid tumour and papillary adenocarcinoma showed $0.9 \%$ for each) and nonmalignant group comprised $36.75 \%$ of total studied cases (inflammatory reactions were considered pneumonia depending on clinical correlations and consequent improvement that represented $20.5 \%$ followed by pulmonary tuberculosis $6.8 \%$ ). First sample failed to obtain diagnosis in $32.47 \%$ of cases. Of the 38 negative cases pneumonia accounted for $47.4 \%$ and pulmonary tuberculosis and sarcoidosis $5.3 \%$ for each. In our research the size of the lesions was secluded as once the lesion was radiologically visible, they were considered objective for biopsy.

Many internists are reluctant to accept a diagnosis of nonspecific fibroinflammatory disease established by TBLB because of a lack of data comparing the technique to open lung biopsy. However, the frequent diagnosis of interstitial fibrosis and inflammation noted in many reported studies is similar to reports of open, [26] and standard bronchoscopic lung biopsies, [12]. According to Claude, Smith et al, 1977, if 
the results of TBLB do not support the clinical impression, then an open lung biopsy must be performed, [27].

Using ultrasound guided TBLB in our research stands for an important supporting tool to avoid blind biopsy trials with high risk for negative yield and necessity for procedure repetition with more frequent complications. The attempts at blind biopsy of solitary peripheral lesions were disappointing The blind biopsy was the least successful, and even transbronchial biopsy under fluoroscopic guidance was less rewarding than percutaneous aspiration needle biopsies done by Grech et al. 1978; and Grech, 1976, [28, 29]. For more sincerity, in one more study by Clack et al., 1977 experience, blind biopsy of peripheral coin lesions has little place, [30] Other workers have encompassed greater success in taking biopsy specimens from these lesions using fluoroscopic guidance (Hanson et al. (1976) $71 \%$ and Ellis (1975) up to $81 \%$ success, depending on the size of the lesion), [31, 32].

There is limited information on the optimal number of transbronchial biopsies. In general, four to six biopsy specimens are adequate in majority of patients with diffuse lung disease, [6]. In one study, $53 \%$ of diagnoses were provided with the first specimen, and $33 \%$ of diagnoses were provided with the second specimen, [33]. In patients with stage II and III sarcoidosis 4-6 TBLB specimens provide optimal diagnostic yield, [34]. However, for stage I sarcoidosis up to ten biopsy specimens may be needed for maximum diagnostic yield, [35]. In our study; sarcoidosis required two sessions to be diagnosed. For localized peripheral lung mass and lung infection, a minimum of 6 and if feasible, up to 10 biopsy specimens should be obtained, [36]. In a large study, the diagnostic yield from localized lesions increased from $23 \%$ with $1-3$ specimens to $73 \%$ with 6-10 specimens, [6]. In our study ultrasound guided TBLB was conducted for 3 specimens taking in the first session that may reduced the succeeded cases performed from the first time. From the first session, the transthoracic US guided TBLB succeed in the diagnosis of $69.69 \%$ of cases underwent the procedure on the other hand blind TBLB (cTBLB) succeed in the diagnosis of $66.7 \%$. However, by the second session, transthoracic US guided TBLB achieved diagnosis in $81.81 \% \%$ and blind TBLB achieved the diagnosis in $71.43 \%$. The overall diagnostic yield of TBLB was $74.4 \%$ ( 87 cases out of $117 ; 79$ cases by the first TBLB and 8 cases by second TBLB).

TBLB succeeded in the diagnosis of 79 cases out of 117 cases accomplished in our study with success rate $(67.52 \%)$ and failed in 38 cases. Follow up of these cases was considered for two weeks; 18 cases proved to be inflammatory consolidation and improved with nonspecific treatment and according to the distance from the chest wall, 12 cases underwent $\mathrm{CT}$ guide true cut biopsy and proved to be: 2 cases due to pulmonary TB 6 cases due to squamous cell carcinoma and 4 cases due to pulmonary Hodgkin lymphoma but 6 cases diagnosed to be adenocarcinoma and 2 cases due to sarcoidosis by a second TBLB. In the current research, the size and site of the lesion were outside our scope as all lesions with negative endobronchial findings and could be assessed by transthoracic ultrasound were enrolled in this study.

Owing to Mazzone et al, 2002, even though TBLB provided adequate lung specimen in $70-90 \%$ of cases, a specific diagnosis could not be rendered in a significant proportion of the patients. TBLB is the most useful sampling method for the diagnosis of peripheral lung cancer. The average diagnostic yield from TBLB is $57 \%$ with a range of $17-77 \%$ in patients with peripheral lung cancers, [37] The diagnostic yield of TBLB for lung cancer increases with the number of biopsy specimens. In one study, the diagnostic yield was $21 \%$ when 1-3 TBLB specimens were obtained and $78 \%$ when 6-10 specimens were obtained, [6]. In another study, the diagnostic yield increased from $45 \%$ with the first specimen to $70 \%$ with multiple TBLB specimens, [36]. Pooled data from ten studies shows a diagnostic yield of $34 \%$. TBLB provides rapid diagnosis in $17-60 \%$ of cases with confirmed active tuberculosis, [38- 41] and is the exclusive source of diagnostic specimen in 10-20\% of these patients, [42-44]. For example, in one study, TBLB provided diagnosis in $57 \%$ of patients with community-acquired pneumonia who failed antimicrobial therapy, [45]. TBLB specimens can demonstrate mycobacterial or fungal infection, and may provide tissue diagnosis of other conditions that can mimic pneumonia such as bronchioloalveolar cancer, cryptogenic organizing pneumonia, and hypersensitivity pneumonitis. In cavitary lesions, obtaining biopsies from the wall of the cavity increases the chances of detecting underlying lung pathology. In Salzman et al., work 1992; microbiological analysis was limited to detect gram positive and gram negative bacterial culture. Traditional flexible bronchoscopy with TBLB has a low diagnostic yield in these patients. For the lung nodules $<2 \mathrm{~cm}$, the data from ten studies shows the average diagnostic yield of FOB to vary from 11 to $76 \%$ with an average of $34 \%$, [46] On multivariate analysis, the independent predictors of obtaining diagnostic tissue sample from lung nodules with flexible bronchoscopy in a study were malignant etiology, and a positive bronchus sign, again reinforcing the importance of size and bronchus sign as important determinants of diagnostic yield of conventional bronchoscopy in these patients, [47]. There are several problems with the use of CTfluoroscopy with conventional bronchoscopy. The ability to visualize the lesion does not guarantee that the operator will be able to advance the biopsy instrument to the target. Also, performing bronchoscopy in CT room poses major scheduling and logistic difficulties. Bronchoscopy is often an awkward experience both for the patient and the operator, and the CT room may be ill-equipped to manage a serious bronchoscopy related complications. Perhaps the most important drawback is the risk associated with excessive radiation exposure to the patients and the operators. According to current estimates, the radiation exposure to the patients with CT- fluoroscopy is 2-5 times that of conventional fluoroscopy, [48, 49], so transthoracic ultrasound pretenses a diagnostic safety shelter for both operator and medical staff and patients but in most cases take 
15 minutes that need more patients care and necessitate affordable general condition.

An important means of diagnosis is endobronchial ultrasound EBUS TBLB. In a study by Disayabutr et al, 2010, the pulmonary lesions were visible on EBUS image in $98.7 \%$ of cases. The overall diagnostic yield of EBUS-TBLB was $66.4 \%$. The diagnostic yield in the infiltrative and mass lesions was $86.4 \%$ and $63.1 \%$, respectively. The lesions which EBUS probe located within it were diagnosed by EBUS-TBLB about $74.8 \%$. The benign and malignant lesions were diagnosed by EBUS-TBLB about $81.1 \%$ and $58.6 \%$, respectively. The average EBUS time was $3.55+/-$ 2.29 minutes. No complication of EBUS and transbronchial lung biopsy were observed in their study, [50].

Although no major complications were encountered in a series by Zavala of 40 patients, certain precautions are essential, [51]. Suction must be used sparingly to avoid hypoxemia, [52] and supplemental oxygen should be available during the procedure. In a collected series of 438 patients, the, incidence of bleeding was $9 \%,[51]$. If the coagulation mechanism is significantly altered, then specific therapy must be instituted prior to biopsy to prevent serious hemorrhage. An open lung biopsy should be the primary diagnostic procedure in the presence of an uncorrected bleeding diathesis. Out of the 117 cases in our work, 5 cases developed complications (4.2\%) that were in the form of residual pneumothorax in 3 patients, $2.56 \%$ ( 2 in malignant group and one in nonmalignant group) and haemoptysis in two patients $1.7 \%$ (one in each group) that was controlled by haemostatics and did not need surgical intervention. No complications were detected from manipulation of negative cases either by $\mathrm{CT}$ guided biopsy or rebiopsy by TBLB. cTBLB presented $2.56 \%$ of cases while TTUS-TBLB accounted for $1.7 \%$ of complications encountered. For this novel approach US guided transbronchial lung biopsy there were some limitations presented in obese stocky patients, patients with chest wall deformity, lesions in inaccessible sonographic areas as subscapular or apical lesions in addition to presence of diffuse pleural thickening, organized pleural effusion localized pneumoyhorax or parenchymal lesions as bullous lung disease that impede sonographic waves to be transmitted and weigh down the echographic access to the lesion else more.

\section{Conclusions}

Transthoracic ultrasound guided TBLB is a prevailing tool for diagnosis when applied with realistic expectations on the part of both pulmonologists and pathologists and knowledge of the diseases and conditions that should be successfully biopsied. This technique can substitute endobronchial ultrasound (EBUS), CT or fluoroscopic guided assistance especially with appropriate patients' selection to undergo biopsy and in the accurate interpretation of different pulmonary lesions. Nonneoplastic as well as neoplastic conditions most commonly diagnosed by means of this procedure. Close interaction between the clinical triad of pulmonologist, radiologist, and pathologist augments the utility of the transthoracic ultrasound TBLB and improves the quality of patient care. The results of real-time TTUSTBLB were promising and may improve the overall diagnostic yield of TBLB in either benign or malignant pulmonary shadows.

\section{References}

[1] Leoncini B and Palatresi R. Transbronchial lung biopsy (a technical contribution). Dis Chest. 1968; 53:736-742.

[2] Smart J. Transbronchial pulmonary biopsy. Thorax. 1966; 21: 444.

[3] Churg A and Schwarz M. Transbronchial biopsy and usual interstitial pneumonia: a new paradigm? Chest. 2006; 129:1117-1118.

[4] Berbescu EA, Katzenstein AL, Snow JL and Zisman DA. Transbronchial biopsy in usual interstitial pneumonia. Chest. 2006; 129: 1126-1131.

[5] Wu CC, Maher MM and Shepard JA. Complications of CTguided percutaneous needle biopsy of the chest: prevention and management. AJR Am J Roentgenol. 2011; 196:678-82.

[6] Descombes E, Gardiol D and Leuenberger P. Transbronchial lung biopsy: an analysis of 530 cases with reference to the number of samples. Monaldi Arch Chest Dis. 1997; 52:324-9.

[7] Andersen HA. Transbronchial lung biopsy for diffuse pulmonary disease. Results in 939 patients. Chest. 1978; 73:734S-6.

[8] Ellis JH. Transbronchial lung biopsy via the fiberoptic bronchoscope. Experience with 107 consecutive cases and comparison with bronchial brushing. Chest. 1975; 68:524-32.

[9] Hanson RR, Zavala DC, Rhodes ML, Keim LW and Smith JD. Transbronchial biopsy via flexible bronchoscope: results in 164 patients. Am Rev Respir Dis. 1976; 114: 67-72.

[10] Mitchell DM, Emerson CJ, Collins JV and Stableforth DE. Transbronchial lung biopsy with the fiberoptic bronchoscope: analysis of results in 433 patients. Br J Dis Chest. 1981;75: 258-62.

[11] Zellweger JP and Leuenberger PJ. Cytologic and histologic examination of transbronchial lung biopsy. Eur J Respir Dis. 1982; 63: 94-101.

[12] Andersen HA and Fontana RS: Transbronchoscopic lung biopsy for diffuse pulmonary diseases: technique and results in 450 cases. Chest. 1972, 62:125

[13] Levin DC, Wicks AB and Ellis JH: Transbronchial lung biopsy via the fiberoptic bronchoscope. Am Rev Respir Dis. 1974, 110:4

[14] Scheinhorn DJ, Joyner LR and Whitcomb ME: Transbronchial forceps lung biopsy through the fiberoptic bronchoscope in Pneumocystis carinii pneumonia. Chest. 1974 66:294

[15] Prasoon Jain, Sarah Hadique, and Atul C. Mehta, Transbronchial Lung Biopsy A.C. Mehta and P. Jain (eds.), Interventional Bronchoscopy: A Clinical Guide, Respiratory Medicine 10. 2013, Springer Science and Business Media New York 
[16] Asano F, Aoe M, Ohsaki Y, et al. Deaths and complications associated with respiratory endoscopy: a survey by the Japan Society for respiratory endoscopy in 2010 . Respirology. 2012; 17(3): 478-85.

[17] Pue CA, Pacht ER. Complications of fiberoptic bronchoscopy at a university hospital. Chest. 1995; 107:430-2.

[18] Ahmad M, Livingston DR, Golish JA, Mehta AC, Wiedemann HP. The safety of outpatient bronchoscopy. Chest. 1986; 90:403-5.

[19] Milman N, Fourschou P, Munch EP, Grode G. Trsnbronchial lung biopsy through fi beroptic bronchoscope. Results and complications in 452 examinations. Respir Med. 1994; 88:749-53.

[20] Cunningham JH, Zavala DC, Corry RJ and Keim LW. Trephine air drill, bronchial brush, and fiberoptic transbronchial lung biopsies in immunosuppressed patients. Am Rev Respir Dis. 1977; 115:213-20.

[21] Jain P, Sandur S, Meli Y, Arroliga AC, Stoller JK and Mehta AC. Role of flexible bronchoscopy in immunocompromised patients with lung in filtrates. Chest. 2004; 125:712-22.

[22] White P, Bonacum JT and Miller CB. Utility of fiberoptic bronchoscopy in bone marrow transplant patients. Bone Marrow Transplant. 1997; 20:681-7.

[23] Lai R-S. , Lee S. S-J., Ting Y-M., Wang H-C., Lin C-C. and $\mathrm{Lu}$ J-Y. Diagnostic value of transbronchial lung biopsy under fluoroscopic guidance in solitary pulmonary nodule in an endemic area of tuberculosis. Respiratory Medicine,1996, 90, $139-143$

[24] Cortese DA and McDougall JC. Biopsy and brushing of peripheral lung cancer with fluoroscopic guidance. Chest. 1979; 75: 141-145.

[25] Radke JR, Conway WA, Eyler WR et al. Diagnostic accuracy in peripheral lung lesions: Factors predicting success with flexible fiberoptic bronchoscopy. Chest. 1979; 16: 176-179.

[26] Ray JF, Lawton BR, Myers WO, et al: Open pulmonary biopsy: nineteen-year experience with 416 consecutive operations. Chest. 1976, 69:43

[27] Claude W. Smith, M.D., Gordon F. Murray, M.D., Benson R. Wilcox, M.D., Peter J. K. Starek, M.D., and David J. Delany, M.D. The Role of Transbronchial Lung Biopsy in Diffuse Pulmonary Disease The AM\& of Thoracic Surgery. 1977, Vol 24 No 1 July

[28] Grech, P., Gray, P. B., Lambourne, C. A., Clark, R. A., and Townshend, R. H. Aspiration needle biopsy for solitary, peripheral lung lesions. British Journal of Diseases of the Chest. 07/1978; 51(606):432-6.

[29] Grech P. Aspiration lung biopsy (abstract). British Journal of Diseases of the Chest, 1976, 70, 284.

[30] Clark R. A., Gray P. B., Townshend R. H. and Howard P. Transbronchial lung biopsy: a review of 85 cases. Thorax, 1977, 32, 546-549

[31] Hanson, R. R., Zavala, D. C. Rhodes, M. L., Keim, L. W., and Smith, J. D. Transbronchial biopsy via flexible fiberoptic bronchoscope: results in 164 patients. American Review of Respiratory Diseases, 1976, 114, 67-72.

[32] Ellis J. H. Transbronchial lung biopsy via the fibreoptic bronchoscope. Chest, 1975, 68, 524-532.

[33] Curley FJ, Johal JS, Burke ME and Fraire AE. Transbronchial lung biopsy. Can specimen quality be predicted at the time of biopsy? Chest. 1998; 113:1037-41.

[34] Gilman MF and Wang KP. Transbronchial biopsy in sarcoidosis. An approach to determine the optimal number of biopsies. Am Rev Respir Dis. 1980; 122: 721-4.

[35] Rothe RA, Fuller PB, Byrd RB, et al. Transbronchial lung biopsy in sarcoidosis. Optimal numbers and sites for biopsy. Chest. 1980; 77:400-2.

[36] Popovich Jr J, Kvale PA, Eichenhorn MS, Radke JR, Ohorodnik JM and Fine G. Diagnostic accuracy of multiple biopsies from flexible fiberoptic bronchoscopy. A comparison of central versus peripheral carcinoma. Am Rev Respir Dis. $1982 ; 125: 521-3$.

[37] Mazzone P, Jain P, Arroliga AC and Matthay RA. Bronchoscopic and needle biopsy techniques for diagnosis and staging of lung cancer. Clin Chest Med. 2002; 23:137-58.

[38] Wallace JM, Deutsch AL, Harrell JH and Moser KM. Bronchoscopy and transbronchial biopsy in evaluation of patients with suspected active tuberculosis. Am J Med. 1981; 70:1189-94.

[39] Danek SJ and Bower JS. Diagnosis of pulmonary tuberculosis by flexible fiberoptic bronchoscopy. Am Rev Respir Dis. 1979; 119:677-9.

[40] Charoenratanakul S, Dejsomritrutai W and Chaiprasert A. Diagnostic role of fiberoptic bronchoscopy in suspected smear negative pulmonary tuberculosis. Respir Med. 1995; 89:621-3.

[41] Tamura A, Shimada M, Matsui Y, et al. The value of fi beroptic bronchoscopy in culture positive pulmonary tuberculosis patients whose pre-bronchoscopic sputum specimen were negative both for smear and PCR analyses. Intern Med. 2010; 49:95-102.

[42] Kennedy DJ, Lewis WP and Barnes PF. Yield of bronchoscopy for the diagnosis of tuberculosis in patients with human immunodeficiency virus infection. Chest. 1992; 102:1040-4.

[43] Chan CHS, Chan RCY, Arnold M, Cheung H, Cheung SW and Cheng AFB. Bronchoscopy and tuberculostearic acid assay in the diagnosis of sputum negative pulmonary tuberculosis: a retrospective study with the addition of transbronchial biopsy. Q J Med. 1992; 82:15-23.

[44] Salzman SH, Schindel ML, Aranda CP, Smith RL and Lewis ML. Role of bronchoscopy in the diagnosis of pulmonary tuberculosis in patients at risk for HIV infection. Chest. 1992; 102:143-6.

[45] Arancibia F, Ewig S, Martinez JA, et al. Antimicrobial treatment failures in patients with community acquired pneumonia: causes and prognostic implications. Am J Respir Crit Care Med. 2000; 162:154-60.

[46] Rivera MP and Mehta AC. Initial diagnosis of lung cancer. ACCP evidence-based clinical practice guidelines. 2 nd edition. Chest. 2007; 132:131S-48.

[47] Rial MB, Delgado MN, Sanmartin AP, et al. Multivariate study of predictive factors for clearly defined lesions without visible endobronchial lesions in transbronchial biopsies. Surg Endosc. 2010; 24: 3031-6. 
[48] White CS, Weiner EA, Patel P and Britt J. Transbronchial needle aspiration. Guidance with CT fl uoroscopy. Chest. $2000 ; 118: 1630-8$.

[49] Ost D, Shah R, Anasco E, et al. A randomized trial of CT- fl uoroscopic guided bronchoscopy vs conventional bronchoscopy in patients with suspected lung cancer. Chest. 2008; 134:507-13.

[50] Disayabutr S1, Tscheikuna J, Nana A.: The endobronchial ultrasound-guided transbronchial lung biopsy in peripheral pulmonary lesions. J Med Assoc Thai. 2010 Jan; 93Suppl 1:S94-101.

[51] Zavala DC: Pulmonary hemorrhage in fiberoptic transbronchial biopsy. Chest. 1976, 50:584,

[52] Blasco LH, Hernandez IMS, Garrido VV, et al. Safety of the transbronchial biopsy in outpatients. Chest. 1991; 99:562-5. 